\title{
BLINDAGE DE LA GALERIE DE VIDANGE DU BARRAGE DE ROSSENS ISUISSEI
}

THE IRON LINED EVACUATION GALLERY OF THE ROSSENS DAM (SWITZERLAND)

\section{J. TRÜB}

Ingénieur Diplômé E. P. Z

English synopsis p. 226

Le barrage de Rossens, construit par les Entreprises Electriques Fribourgeoises, à Fribourg, et situé sur la Sarine, à peu près à michemin entre Fribourg et Bulle, a été achevé en automne 1948. Le bassin naturel d'alimentation

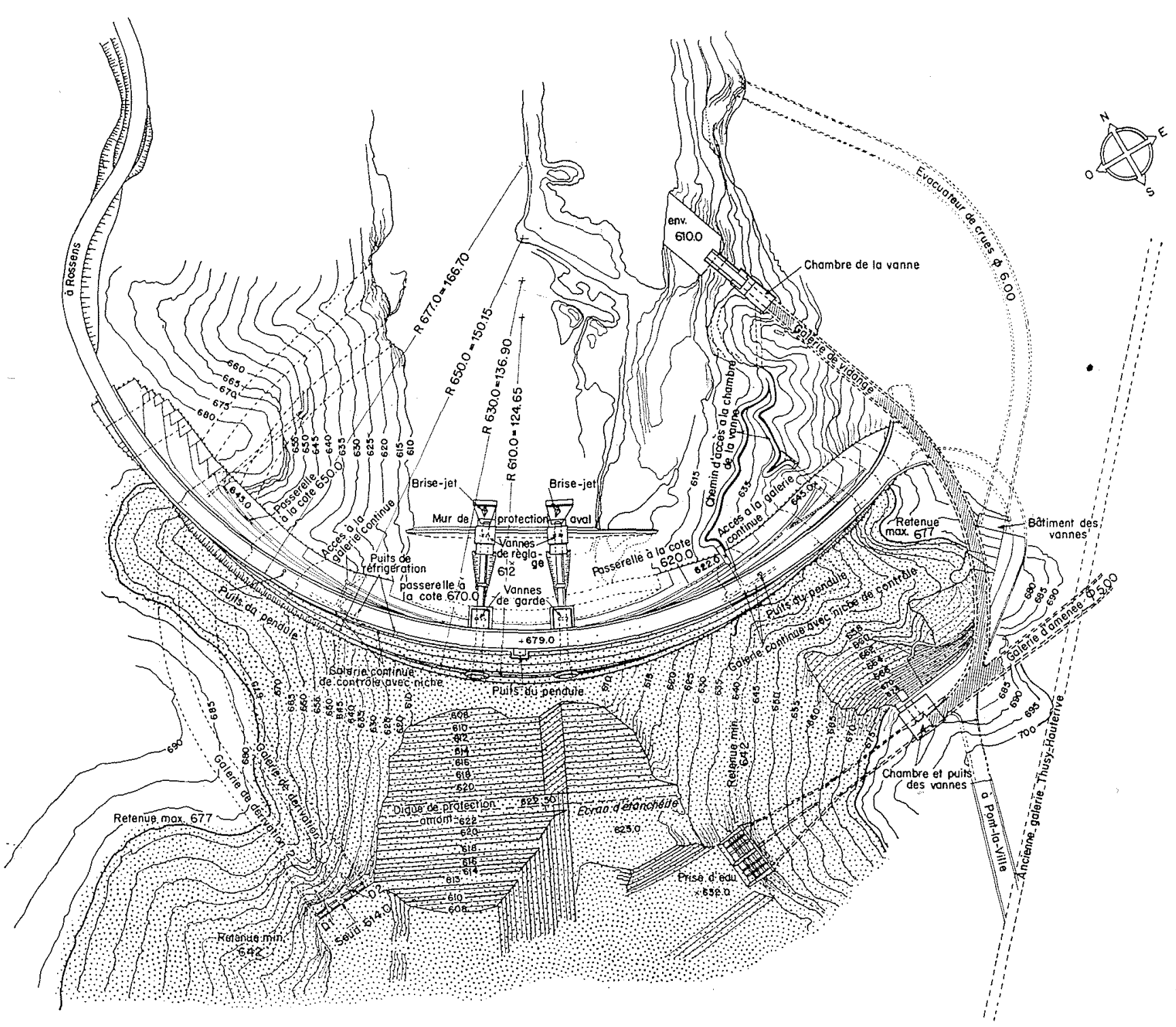

fig. 1 de cette rivière est très étendu et ne comprend que fort peu de glaciers. De ce fait, le régime de la Sarine est éminemment torrentiel. Des crues très importantes se produisent à toute époque de l'année: au printemps à la fonte des

Plan de situation (aimablement W.s à notre disposition par les E. E. F.) 
neiges, en été par temps d'orage, en automne au moment des fortes pluies et en hiver lors des coups de fohn. Le débit d'étiage est de l'ordre de grandeur de $5 \mathrm{~m}^{3} / \mathrm{sec}$. tandis que, par exemple, lors de la crue exceptionnelle du 23 novembre 1944, le débit a passé en moins de 24 heures, de 75 à $570 \mathrm{~m}^{3} / \mathrm{sec}$. Ces chiffres donnent une idée de l'importance des crues et de leur rapidité. Bien que le barrage de Rossens ait principalement pour but d'accumuler les crues et de régulariser ainsi le débit de la Sarine au profit des usines hydroélectriques situées en aval et en particulier de celle d'Hauterive, directement alimentée par le lac ainsi créé, il fallait cependant munir le barroge d'ouvrages capables d'évacuer intégralement les crues pour le cas où elles surviendraient inopinément lorsque le lac serait déjà à son niveau maximum. L'eau ne devant pas se déverser par-dessus le barrage, le principal de ces ouvrages est l'évacuateur de crues, voir figure 1, situé sur la rive droite à quelques mètres en dessous de la crête du barrage et pouvant débiter au maximum $350 \mathrm{~m}^{3} / \mathrm{sec}$. Lorsque cet évacuateur s'avère insuffisant, il est secondé soit par les 2 conduites de vidange placées au pied du barrage, d'un débit de $140 \mathrm{~m}^{3} / \mathrm{sec}$. chacune, soit par la galerie de vidange branchée sur la galerie d'amenée à l'usine d'Hguterive et capable à elle seule d'assurer un débit de $270 \mathrm{~m}^{3} / \mathrm{sec}$.
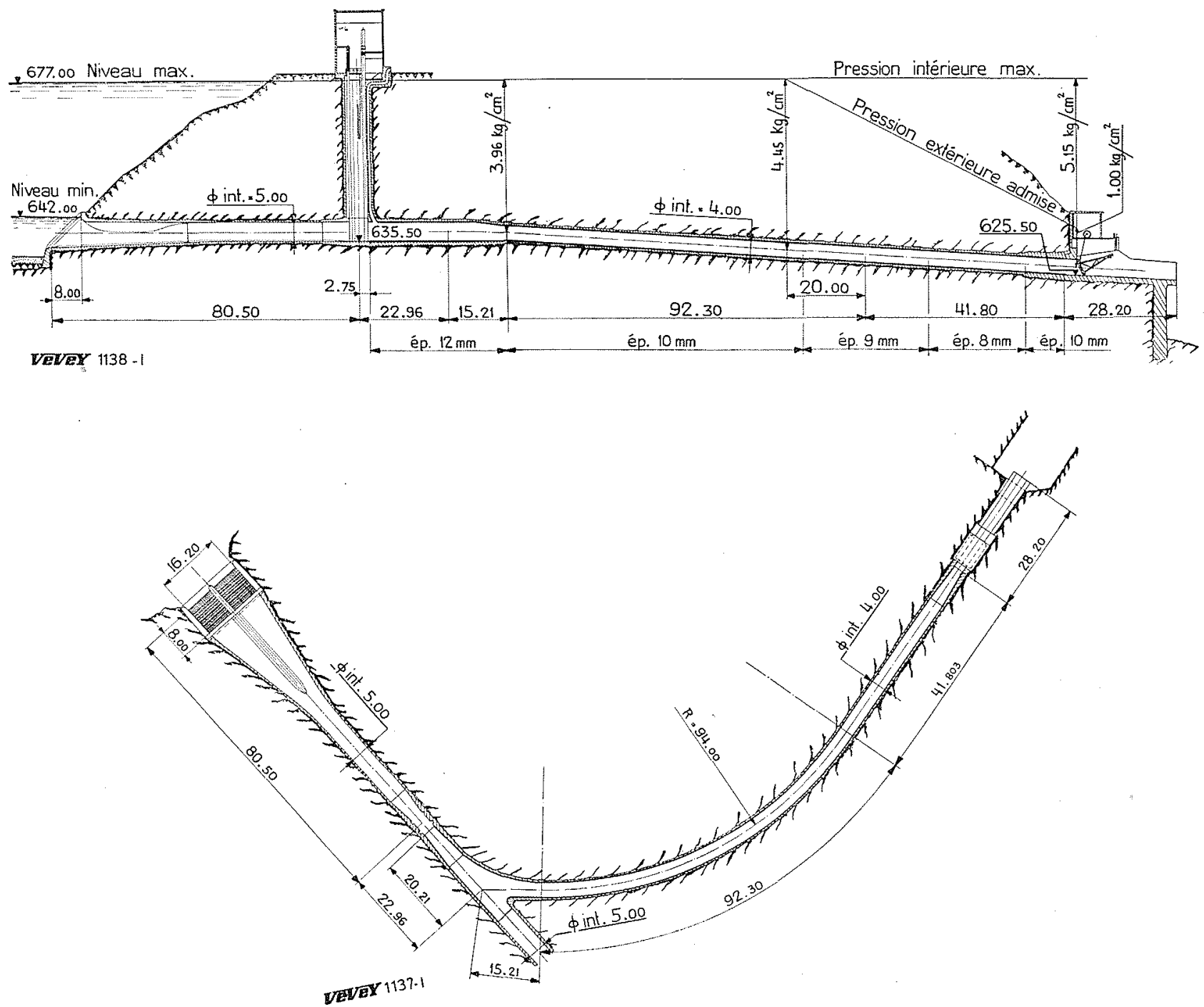

fig. 2

Plan et élévation de la galerie de vidange, avec diagrammes des pressions intérieures et extérieures servant de base au calcul 

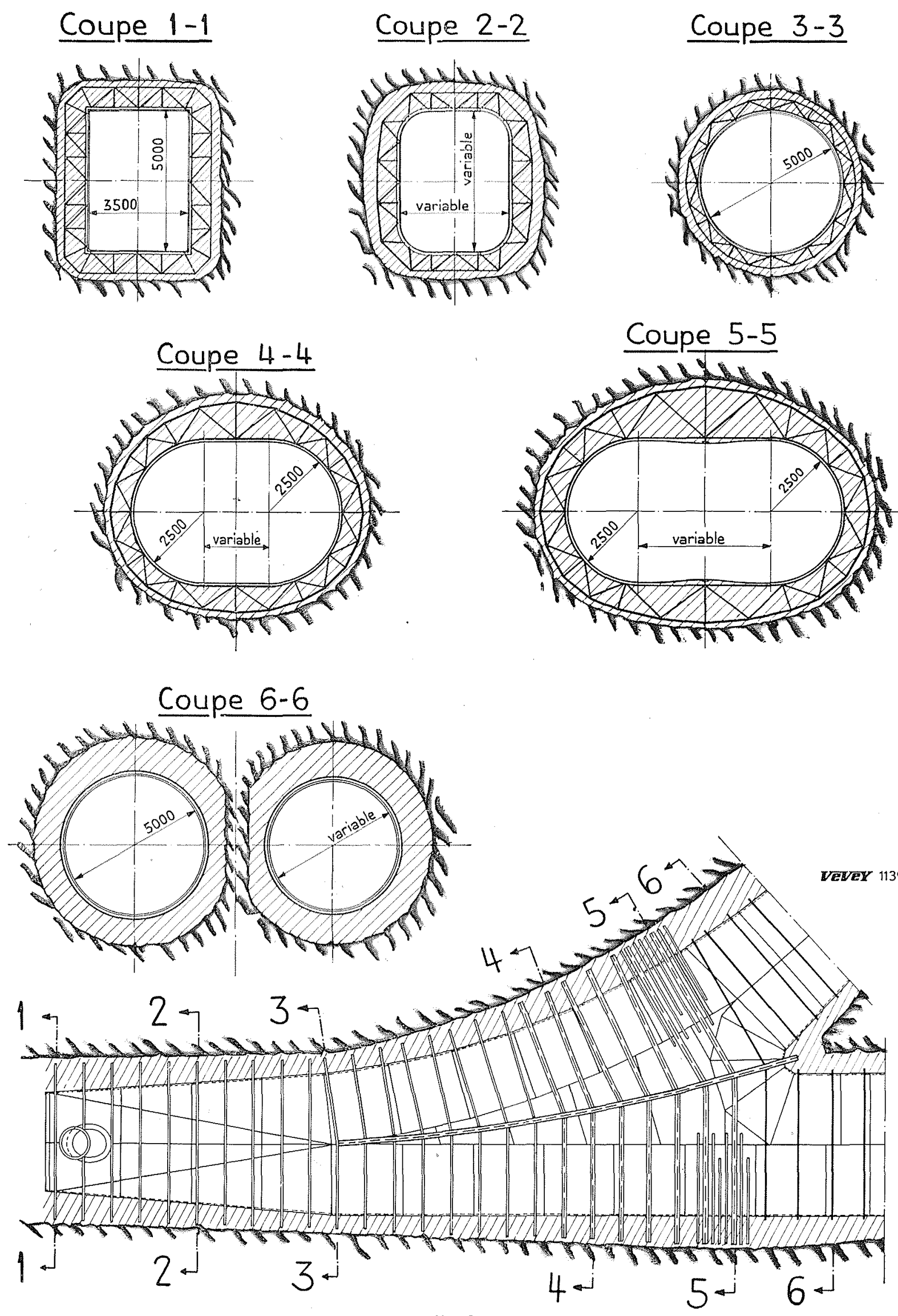

fig. 3

Coupes longitudinale et transversales de la culotte.

\section{Coupe 3-3}
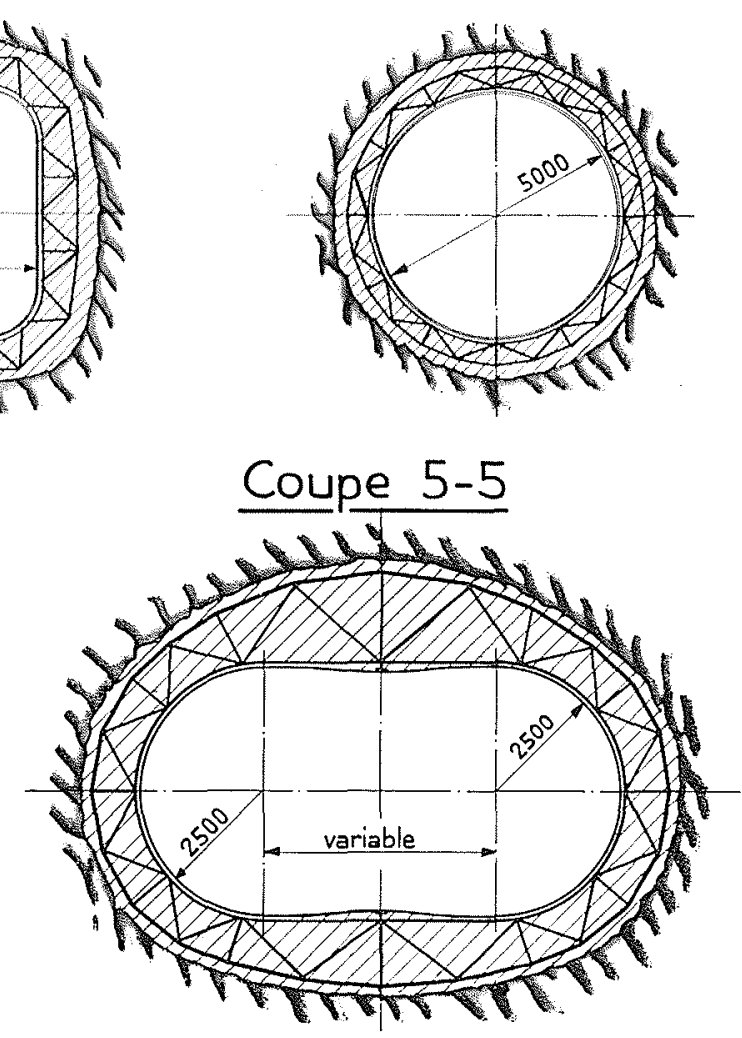
Le but de cet article est de donner une description détaillée du blindage de cette galerie de vidange, dont la fourniture a été assurée par les soins de VEVEY.

\section{Généralités.}

La galerie de vidange ayant un diamètre de $4 \mathrm{~m}$., la vitesse maximum de l'eau est de l'ordre de grandeur de $22 \mathrm{~m}$./ $\mathrm{sec}$. Comme elle est percée dans la molasse et qu'elle se trouve à proximité du barrage, on comprendra sans peine pourquoi il a fallu la blinder sur toute sa longueur.

Le lot attribué à VEVEY commence à partir des vannes de sécurité installées au. fond d'un puits pour se terminer à l'entrée de la vanne de vidange débouchant directement dans la Sarine, voir figure 2 .
La galerie débute par une section rectangulaire d'une largeur de $3 \mathrm{~m} .50$ et d'une hauteur de $5 \mathrm{~m}$. qui se transforme graduellement pour devenir, $10, \mathrm{~m}$. plus loin, une section circulaire de $5 \mathrm{~m}$. de diamètre. Cette partie est commune à la galerie d'amenée et à celle de vidange. A partir de là commence la bifurcation des deux conduites, partie dénommée culotte et dont les diverses sections sont représentées par la $\mathrm{fi}$ gure 3. La branche de droite, rectiligne, fait partie de la galerie d'amenée et a un diamètre de $5 \mathrm{~m}$. La branche de gauche, curviligne, assure la liaison avec la galerie de vidange proprement dite, laquelle décrit en plan un arc de cercle de $94 \mathrm{~m}$. de rayon et ramène ainsi l'eau dans la Sarine. A l'aval, la galerie passe de nouveau de la forme circulaire à la forme rectangulaire pour pouvoir se raccorder à la vanne de vidange. La pente de la galerie est de $6,8 \%$. Son extrémité en aval est à la cote 625 , tandis que le niveau

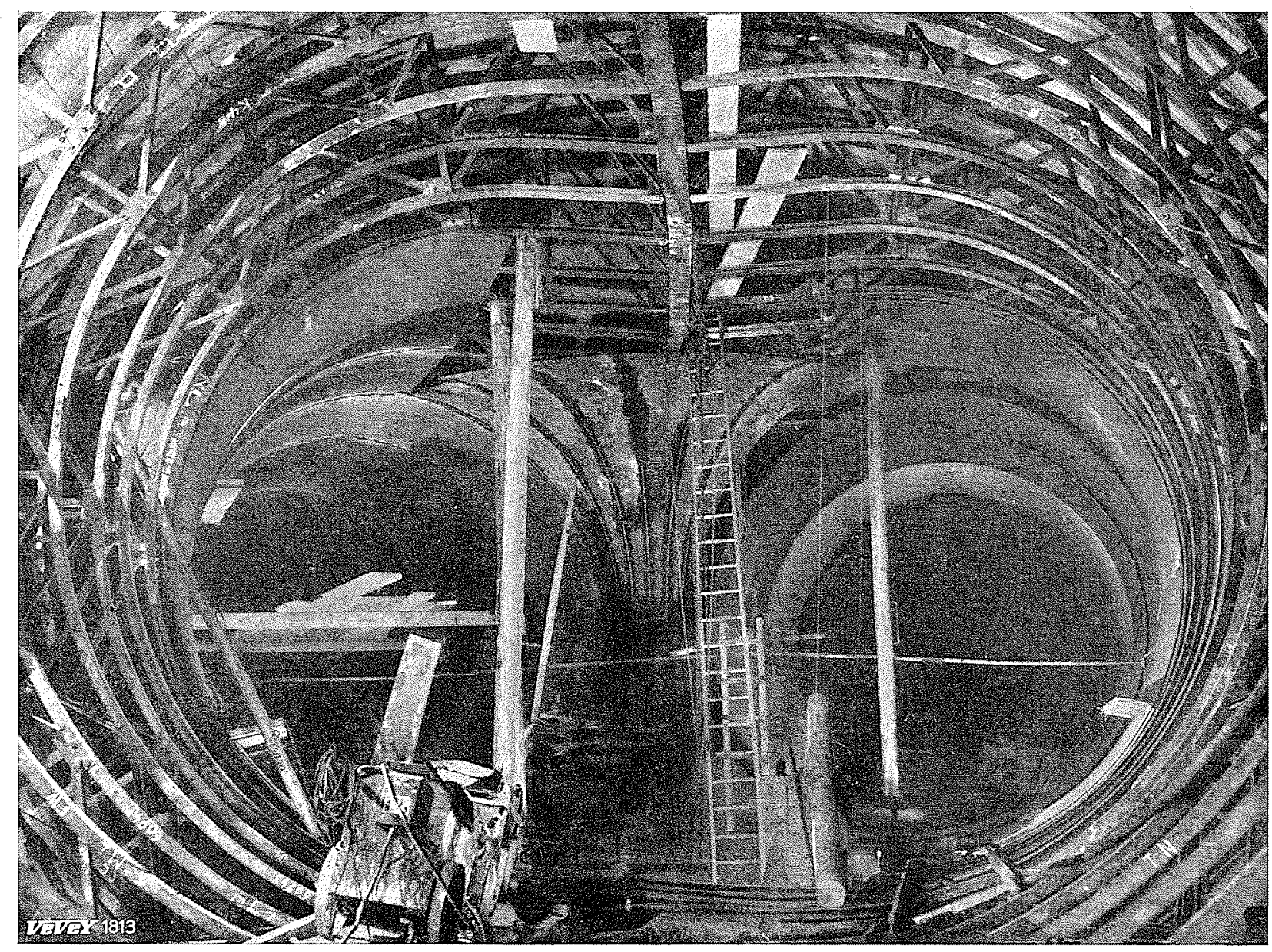

fig. 4

Cintres de la culotte. 
maximum de la retenue est à la cote 677 . La conduite est donc soumise à une pression intérieure correspondant à une colonne d'eau de $52 \mathrm{~m}$. lorsque la vanne de vidange est fermée, ce qui est normalement le cas. Cependant, il fallait aussi envisager le cas inverse, celui qui se présente lorsque la vanne de sécurité en amont est fermée et que la galerie est viciée. Comme celle-ci se trouve dans le voisinage du lac il est possible que le blindage de la galerie soit soumis, par suite d'infiltrations, à une pression extérieure variant de $40 \mathrm{~m}$. d'eau en amont jusqu'à zéro en aval. Les diagrammes de la figure 2 indiquent les valeurs admises pour les pressions intérieures et extérieures servant de base au calcul du blindage.

On conçoit que ces deux cas de charge diamétralement opposés aient soulevé des problèmes rarement rencontrés jusqư'à ce jour, surtout si I'on tient compte des grandes dimensions de l'ouvrage.

L'enveloppe de la galerie est en effet constituée par un blindage en tôle d'acier qui doit être parfaitement étanche, celui-ci est enrobé de béton servant de liaison entre le blindage et la molasse dont le module d'élasticité varie entre 20.000 et $30.000 \mathrm{~kg} / \mathrm{cm}^{2}$.

Le principal problème à élucider était donc de déterminer la répartition des efforts dus à la pression hydraulique entre le blindage en acier, le revêtement en béton et la roche molassique. La littérature technique n'est pas encore très riche dans ce domaine (1)

Pour parer aux incertitudes des calculs et aux imperfections inévitables de l'enrobage en béton, il fallait choisir pour le blindage une tôle capable de subir, sans se rompre, d'importantes déformations. On a adopté la qualité $S . M$. chaudière 1 , d'une résistance à la traction de 34 à $42 \mathrm{~kg} / \mathrm{mm}^{2}$ et d'un allongement spécifique de 21,6 à $27 \%$, mesuré sur longue éprouvette.

\section{Culotte et cônes de raccordement.}

Comme l'indique la figure 3 , le blindage de la culotte est entouré d'une épaisseur de béton variant entre 0,60 et $2,00 \mathrm{~m}$. L'espace disponible

(1) Nous prions nos lecteurs que cette question intéresse, de bien vouloir se reporter aux études récemment parues dans le "Bulletin Technique de la Suisse Romande". No 17, 1947 (suite à paraître en été 1948), dans la "Schweizerische Bauzeitung",
$N^{\circ} 14$, Bd. 124, 1944, et dans "Cours d'Eau et Energie", No $11 \% 12,1947$.

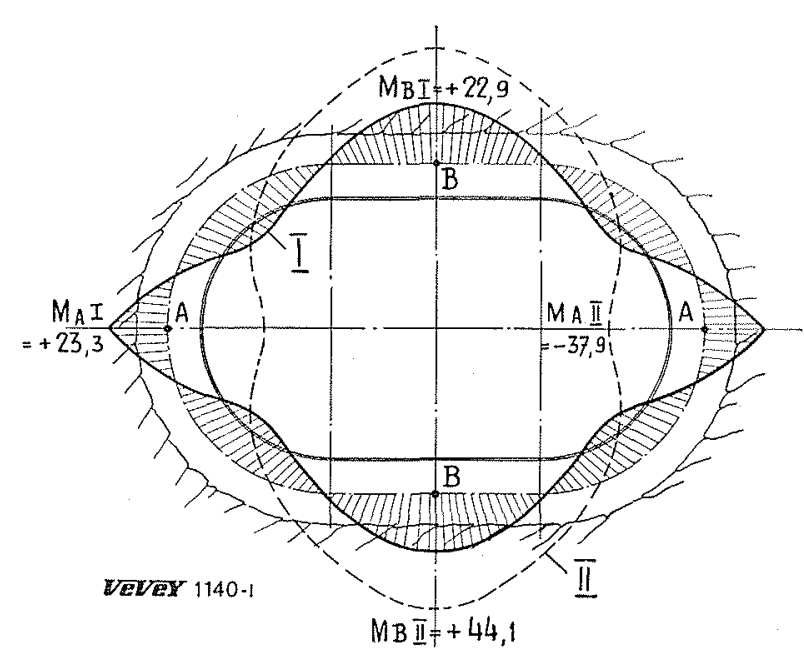

fig. 5

Coupe 5-5 de la culotte (voir fig. 3) avec diagrammes des moments fléchissants d'un cintre transversal, dus à la pression extérieure. Le diagramme I est établi en supposant que la distance A-A est invariable (élargissement empêché par le rocher, par deux réactions

Le diagramme II se rapporte au cas où la déformation du cintre est complètement libre (sans réactions horizontales).

entre la tôle et la roche a permis de munir le blindage de cintres extérieurs, sous forme de poutres triangulées en fers profilés, qui avaient à remplir les deux buts suivants :

1. Servir de gabarit pour le montage des tôles de blindage de grand format, les cintres étant montés en premier lieu dans la galerie, voir figure 4.

2. Armer le béton et créer ainsi une coque rigide en béton armé, résistant aux efforts de compression et de flexion dus tantôt à la pression intérieure, tantôt à la pression extérieure.

La figure 5 montre la courbe admise par calcul des moments fléchissants d'un cintre transversal, caractéristique de la culotte, soumis à la pression extérieure. II est évident que plus la forme du blindage diffère de celle d'un cercle, plus les moments fléchissants sont importants. En plus de ces sollicitations qui intéressent l'enveloppe en béton armé tout entière, il fallait assurer la résistance de la tôle seule à une pression d'eau éventuelle entre tôle et béton. Cette condition a été réalisée par des ancrages en fers plats $50 \times 10 \mathrm{~mm}$. Dans les régions planes de la culotte, la tôle a une épaisseur de $20 \mathrm{~mm}$. et elle est calculée comme supportée par points aux endroits des ancrages. Dans les parties cintrées, l'épaisseur du blindage a pu être réduite à $12 \mathrm{~mm}$. du fait de l'effet de voûte. L'exécution de la culotte a été rendue particu- 


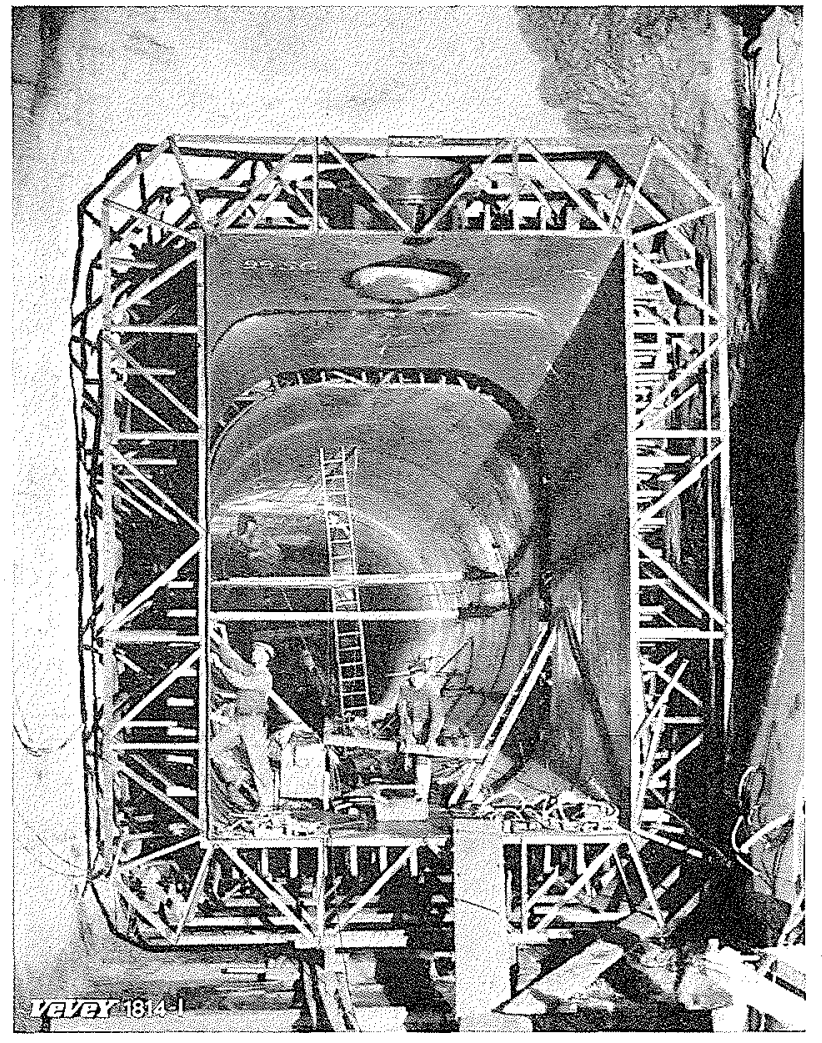

fig. 6

Cône de l'amont en montage.

lièrement compliquée par le fait qu'elle devait réaliser le raccordement de deux conduites en courbe de diamètre différent, l'un de 5 et l'autre de $4 \mathrm{~m}$.

La partie conique, à l'amont de la culotte, a été armée d'une façon analogue. Sa construction est représentée par la figure 6 , vue prise en galerie.

La figure 7 représente le cône de l'aval, raczordant la conduite à la vanne de vidange, de construction semblable à celle de l'autre cone.

\section{Galerie.}

La galerie proprement dite, d'un diamètre constant de $4 \mathrm{~m}$. et d'une longueur totale d'environ $134 \mathrm{~m}$. a été calculée d'après une méthode publiée dans le «Bulletin technique de la Suisse romande », mentionné précédemment. Conformément au diagramme des pressions extérieures (fig. 2), l'épaisseur du blindage varie entre $10 \mathrm{~mm}$. à l'amont, $9 \mathrm{~mm}$. au milieu et $8 \mathrm{~mm}$. à l'aval. La distance des pattes d'ancrage en fers plats $50 \times 10 \mathrm{~mm}$. est de 250 à $300 \mathrm{~mm}$.

\section{Montage.}

Etant donné les grandes dimensions de l'ouvrage, le transport du blindage ne pouvait s'effectuer qu'en pièces détachées. Celui-ci s'est fait par train jusqu'à Bulle et de là par camions. Le travail d'atelier se réduisait donc au chanfreinage et au cintrage des tôles et à la fabrication des cintres. L'assemblage des tôles par soudure électrique, travail le plus important et le plus difficile, a été exécuté sur place. A cet effet, la chambre des vannes, recouvrant le puits de celles-ci, a été provisoirement agrandie et la voie de roulement du pont roulant qui le dessert, prolongée. De cette façon, on a aménagé un atelier de soudure d'une hauteur respectable de $16 \mathrm{~m}$. dans lequel on a pu exécuter, dans les meilleures conditions possibles, I'assemblage et la soudure des viroles. Celles-ci, une fois terminées, étaient descendues dans le puits des vannes au moyen du pont roulant et amenées à pied d'œuvre par une voie Decauville sur laquelle circulait un chariot spécialement construit pour faciliter la mise en place rapide de la virole. Le centrage de deux viroles consécutives était assuré par un anneau extérieur, soudé au préalable

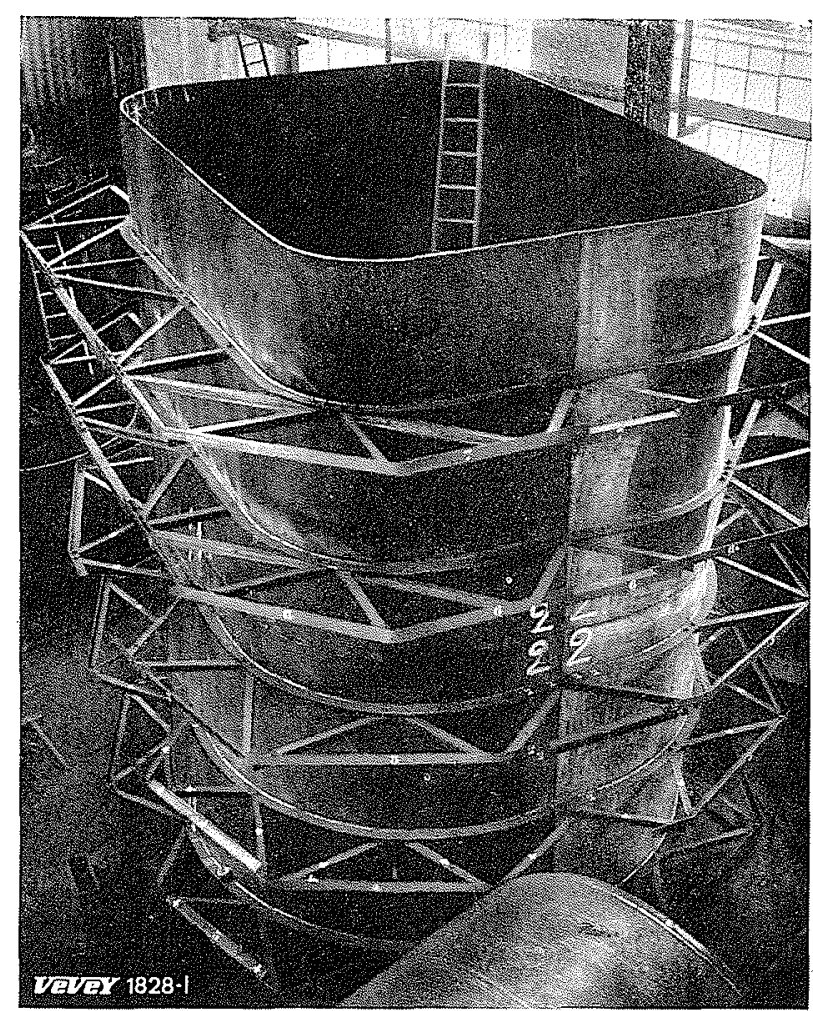

fig. 7

Cône de l'aval assemblé provisoirement en atelier. 


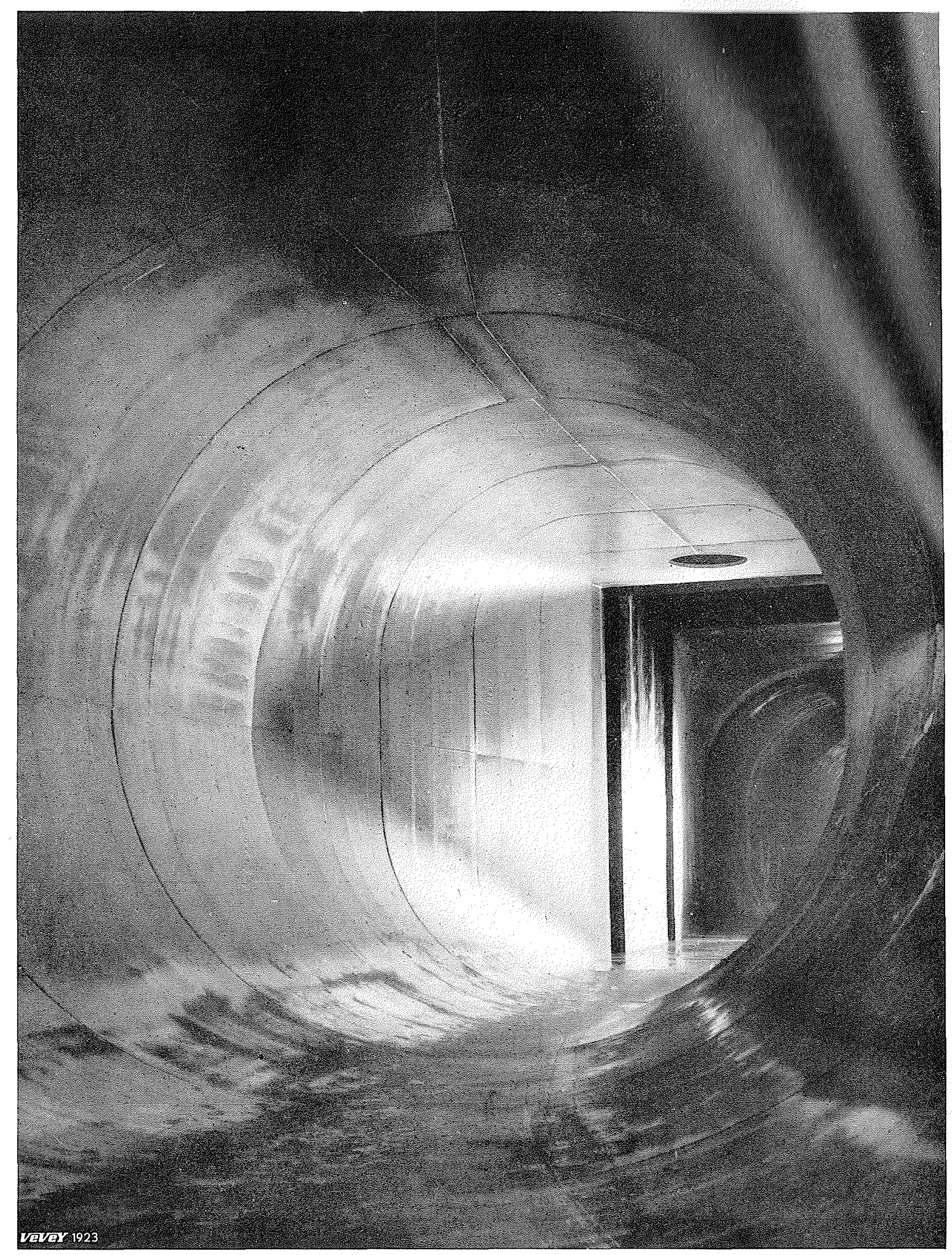

fig. 9

Cône de l'amont; au fond les rainures des vannes de sécurité et le trou d'aération 
à l'extrémité en amont de chaque virole. Pour empêcher la déformation de celle-el pendant son transport, sa mise en place, et son bétonnage, des cintres métalliques extensibles étaient montés et fortement serrés tous les $1 \mathrm{~m} .50$ à I'intérieur de la virole, voir figure 8. Ces cintres, qui n'étaient enlevés qu'après la prise du béton, facilitaient aussi le raccordement des viroles.

Le montage s'est donc fait de l'aval vers l'amont en commençant par le cône de raccordement en aval (figure 7).

Chaque virole de la galerie circulaire était raccordée à la précédente par une soudure circulaire exécutée de l'intérieur seulement, car l'espace libre entre le blindage et le rocher n'était que d'environ $25 \mathrm{~cm}$. Pour la culotte et les cônes de raccordement, cet espace étant beaucoup plus grand, les soudures ont pu être faites de l'intérieur et de l'extérieur. Dès qu'un tronçon d'environ $10 \mathrm{~m}$. était terminé, on procédait à son bétonnage au moyen d'une pompe à béton. Ce travail durait environ 24 heures consécutives pendant lesquelles tous travaux de chaudronnerie étaient rendus impossibles par la présence de la pompe à béton, et des matériaux nécessaires au bétonnage. Pour parfaire au retrait du béton et réaliser une liaison aussi parfaite que possible entre le blindage et la molasse, un mortier de ciment a été injecté sous une pression de $4 \mathrm{~atm}$. au travers de trous filetés, ménagés à cet effet dans la tôle à raison de 3 en moyenne par mètre courant de galerie. Bien entendu, ces injections ont été faites non seulement dans la partie cylindrique de la

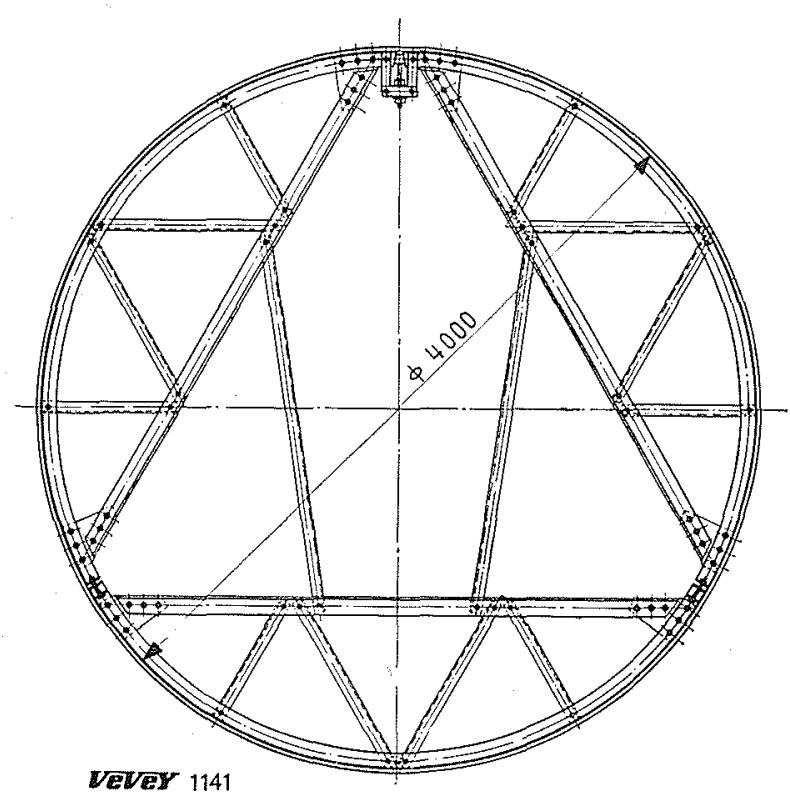

fig. 8

Cintres métalliques provisoires empêchant la déformation du blindage pendant l'assemblage, le transport et le bétonnage.

galerie mais aussi pour la culotte et les deux cônes. Le blindage a parfaitement résisté à cette épreuve sous pression locale.

Pour terminer, mentionnons encore que le maître de l'œuvre a fait nettoyer l'intérieur de la galerie au jet de sable, puis recouvrir le fer ainsi mis à nu de 3 couches de peinture à base de bitume.

(Article reproduit du Bulletin Technique Vevey, 1948), 